\title{
PROGRAMA Chilecalifica: REDES PARA LA ARTICULACIÓN DE LA FORMACIÓN TÉCNICA
}

Los profundos cambios experimentados por Chile en las últimas décadas, especialmente en materia política y económica, le han hecho acreedor a crecientes niveles de reconocimiento de la comunidad internacional, lo que le ha permitido abrir espacios y lograr un posicionamiento auspicioso en el contexto económico regional y mundial.

La estabilidad política y solidez económica han hecho de Chile un lugar atractivo para la inversión internacional, lo que permite ver con optimismo sus posibilidades de desarrollo futuro, en el contexto de una economía globalizada.

Los diversos análisis sobre las posibilidades de inserción exitosa de nuestro país en la economía mundial coinciden en señalar la urgente necesidad de implementar medidas que permitan alcanzar mayores niveles de desarrollo de nuestro capital humano. En esta materia, Chile tiene un notorio déficit, el que resulta evidente si se analiza la cantidad de técnicos con que cuenta, los que están muy por debajo de la proporción requerida, en relación con la cantidad de profesionales que egresa de nuestras universidades cada año.

$\mathrm{Al}$ analizar el escenario nacional, en el cual se debe hacer frente a los desafíos de mejoramiento de los sistemas formativos, es posible detectar ciertos elementos que complican las soluciones factibles. Desde el punto de vista del desarrollo social, los beneficios del crecimiento económico no llegan a todos los chilenos por igual, siendo menos favorecidas aquellas personas de los sectores más pobres y que cuentan con menores niveles de formación. 
El ingreso de las personas y su calidad de vida están directamente relacionados con sus niveles de escolaridad y la calidad de su formación. Si bien, en la medida que aumentan los años de escolaridad de las personas su ingreso es mayor, esta variación sólo comienza a ser significativa a partir de los 12 años de escolaridad. Es decir, las posibilidades de las personas y sus familias de lograr un mayor nivel de bienestar son realmente limitados si no cuentan con las calificaciones y competencias necesarias para una exitosa inserción en el ámbito laboral; en este sentido, la vía de formación técnica se convierte en una de las alternativas válidas para alcanzar y mantener vigentes dichas competencias.

El país ha hecho un esfuerzo importante para reformar la educación técnica que se imparte en el nivel medio, ha venido desarrollando iniciativas de mejoramiento de la calidad y de la equidad en la formación técnica de nivel superior, está ejecutando acciones para mejorar y ampliar la oferta de capacitación laboral y, a partir de 2006, se comenzará la implementación del nuevo marco curricular para la educación de adultos, donde la preparación técnica ocupa un lugar destacado. Por otro lado, se espera acordar un procedimiento para establecer un marco nacional de competencias laborales, el que, además de permitir la construcción de exámenes para evaluarlas y certificarlas, entregará un instrumento para orientar la pertinencia de los currículos de formación técnica.

Aportar a la solución de estos problemas representará uno de los grandes desafíos que deberán enfrentar las instituciones formativas y de capacitación, y que, necesariamente, tendrá que ser abordado en conjunto con los organismos públicos responsables de estos temas. 


\section{El desafío de la formación permanente en Chile}

La creciente velocidad en la generación de conocimientos y su influencia sobre el desarrollo de las sociedades y economías contemporáneas es el sello distintivo del mundo en que nos toca vivir. La capacidad de generación y gestión del conocimiento juega un rol cada día más importante en las posibilidades de desarrollo personal y social, determinando la accesibilidad de los países a los beneficios propios de la economía global.

La gestión de los nuevos conocimientos, la mayor velocidad de obsolescencia de las tecnologías aplicadas en los procesos productivos, la incorporación de nuevas tecnologías de información y comunicación en prácticamente todas las actividades humanas, y la rapidez con que éstas se van actualizando, hacen incuestionable la creciente necesidad de establecer oportunidades de formación permanente de las personas.

La inversión en la formación del capital humano es la mejor vía para el mejoramiento de la calidad de vida de las personas y para alcanzar mayores niveles de desarrollo social y económico en el país.

Para abordar estos temas, Chile asumió el desafío de instalar las bases de un sistema de formación permanente, con el propósito de enlazar armónicamente la educación formal, la capacitación laboral y el reconocimiento de los aprendizajes obtenidos informalmente. Con este fin, el gobierno creó el Programa Chilecalifica, iniciativa conjunta de los ministerios de Economía, Educación y del Trabajo y Previsión Social, que cuenta con el respaldo y cofinanciamiento del Banco Mundial.

La conformación de este sistema implica concertar los esfuerzos del conjunto de instituciones y organizaciones del sector productivo y educativo, para generar ofertas curriculares articuladas y pertinentes, que permitan a las personas desarrollar sus trayectorias formativas de acuerdo con sus necesidades, intereses y capacidades iniciales. 
Para generar mayores oportunidades de formación en un escenario marcado por la constante ampliación del conocimiento y de la innovación tecnológica, el Programa Chilecalifica se ha planteado, entre otras acciones:

a) Atender los requerimientos de formación de técnicos, generando instrumentos y mecanismos que permitan a las personas mejorar los niveles formativos, mantener vigentes sus competencias y ampliar sus alternativas de empleabilidad, con independencia de si ellas provienen de la educación formal, del mundo productivo o si la necesidad les surge de su intención de inserción al ámbito laboral.

b) Desarrollar experiencias que contribuyan a mejorar la calidad de la formación técnica, enfrentando los problemas de conexión entre la formación en oficios, la de técnicos de nivel medio, la de técnicos de nivel superior y la capacitación, además de optimizar la débil conexión existente entre la formación y los requerimientos del mundo productivo y desarrollo regional, y la insuficiente cobertura en la formación de técnicos de nivel superior.

c) Instalar, gradualmente, un marco nacional de competencias laborales e itinerarios de formación técnica y un sistema de información para formación permanente.

\section{Las redes: una estrategia en desarrollo}

Para abordar las deficiencias y problemas que actualmente enfrenta el sistema chileno en la formación técnica, el Programa Chilecalifica ha diseñado líneas de acción, estratégicas y demostrativas, en la perspectiva de aportar a la instalación de las bases de un sistema de formación permanente:

- Proyectos de redes para la articulación de la formación técnica.

- Itinerarios de formación técnica.

- Formación inicial y actualización de profesores y docentes técnicos.

- Sistema de aseguramiento de la calidad de la ofertas de especialidades de enseñanza media técnico-profesional.

Si se quiere lograr un mejoramiento real en la formación de técnicos se necesita la participación de todos los actores 
involucrados en el proceso. Por ello, el Programa Chilecalifica adoptó como estrategia la generación de redes de formación técnica, conformadas por actores relevantes de los sectores productivos, educativos y de la capacitación, organizadas en torno a sectores económicos definidos como prioritarios para el desarrollo por cada una de las regiones.

Las redes, por lo tanto, están integradas por instituciones que imparten educación de adultos, que ofrecen formación técnica en el nivel medio y superior, institutos de capacitación laboral, empresas, organizaciones empresariales y de trabajadores.

Como instrumento para la implementación de esta estrategia, se creó un fondo nacional concursable destinado a financiar proyectos de articulación, con propuestas innovadoras y pertinentes a las necesidades del desarrollo productivo regional, que pongan en funcionamiento ofertas formativas modularizadas con enfoque de competencias laborales, que articulen los distintos niveles y modalidades de formación técnica, incluyendo la capacitación, y que permitan el reconocimiento de los aprendizajes previos.

La organización, coordinación e interacción en red de los actores del mundo productivo y educativo deberá facilitar la articulación de las propuestas formativas de las diferentes modalidades y niveles de formación técnica y capacitación, asegurando que respondan efectivamente a los requerimientos de desarrollo productivo de los sectores económicos prioritarios de las regiones y del país.

Con la implementación de esta estrategia se espera que las redes de articulación de la formación técnica perduren en el tiempo y lleguen a transformarse en un referente regional relevante y dinamizador del desarrollo productivo sectorial. Su consolidación como comunidades de aprendizaje, capaces de actuar en el cambio y la incertidumbre, respondiendo oportunamente al dinamismo de la economía moderna, podría llegar a constituir una verdadera 
garantía de la oportuna y pertinente formación y actualización de los cuadros técnicos que cada región y el país requieran para avanzar en su desarrollo.

El programa propiciará el desarrollo de aproximadamente 42 proyectos de redes, dieciséis de los cuales ya se iniciaron en 2004 y nueve están iniciando sus proyectos en 2005, en diferentes regiones del país y diversos sectores productivos, como se muestra en el mapa.

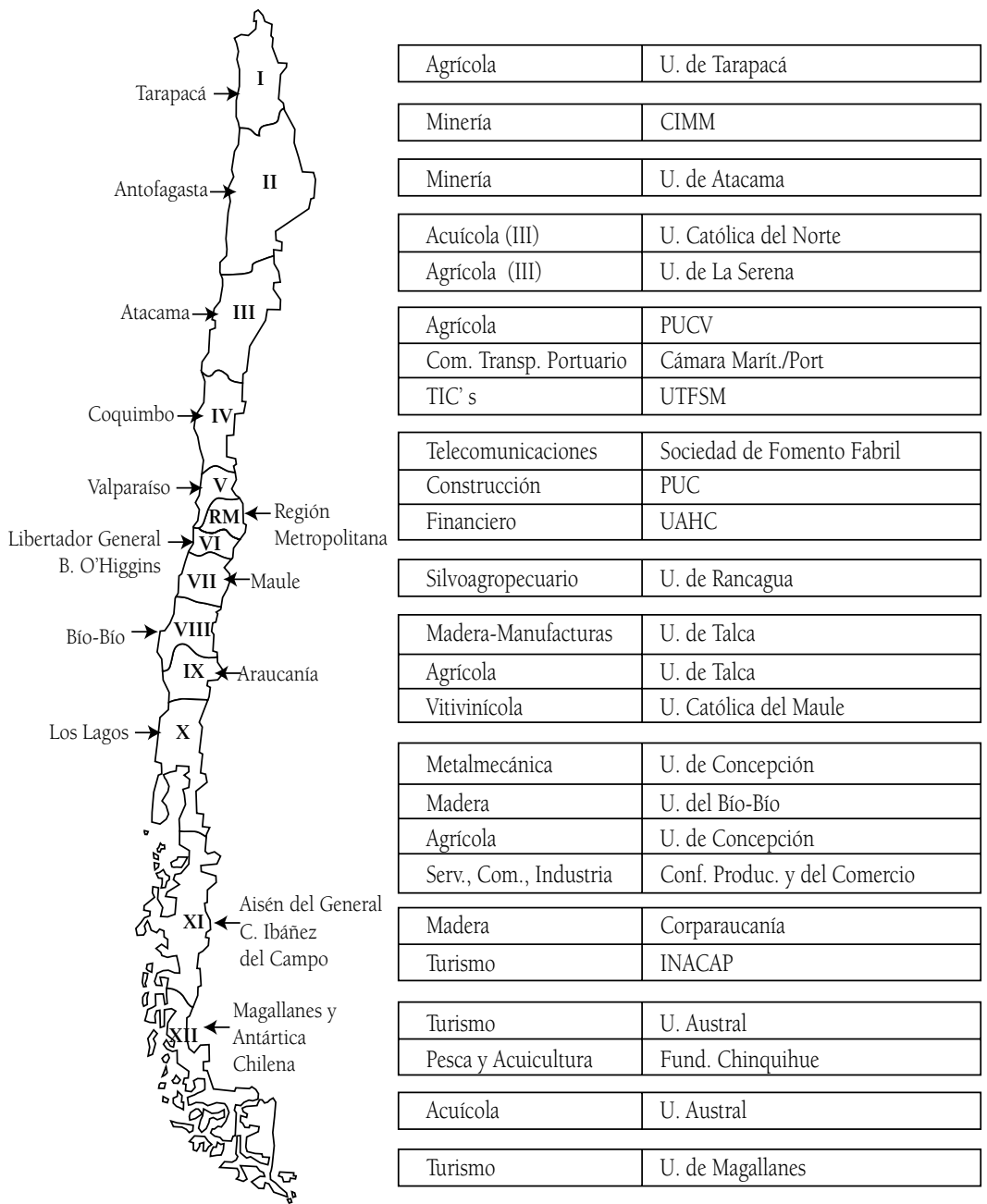


Las redes, como experiencias demostrativas, constituyen verdaderos laboratorios para diseñar e implementar ofertas curriculares articuladas de formación técnica en los sectores económicos y áreas ocupacionales de mayor relevancia para el país.

El desafío que se plantea a las instituciones que conforman las redes no es menor, pues implica un cambio cultural profundo, que tiende a trasladar la base del diseño de las propuestas curriculares desde la oferta formativa a la demanda de técnicos que requiere el país. ¿Qué técnicos se debe formar? ¿Cuál es el perfil profesional que se utilizará como referente para el diseño curricular? ¿Cuáles son las tendencias del desarrollo productivo y la dinámica del empleo a las cuales deben responder los procesos formativos? ¿Cuáles son las capacidades que se debe desarrollar durante el proceso formativo?

La conformación de redes de articulación de la formación técnica implica cambios culturales como los siguientes:

- Que las instituciones formativas acepten que el sector productivo oriente su oferta formativa, al definir las competencias laborales de los técnicos.

- Que se reconozca como válidos los aprendizajes logrados fuera del sistema educativo regular, como son el de la capacitación y la experiencia laboral.

- Que se genere un trabajo colaborativo entre instituciones formativas de los distintos niveles y modalidades, y entre aquéllas y el sector productivo.

Para instituciones que tradicionalmente han tomado sus decisiones en forma independiente y que las implementan con absoluta autonomía, no resulta fácil asumir un trabajo asociado y de toma de decisiones compartidas con instituciones que habitualmente se han considerado competidoras, y que el sector productivo, en muchos casos distante de la entidad formadora, tenga directa injerencia en la definición del tipo de técnicos que ellas deben preparar. 


\section{Los inicios de la colaboración en red}

La misión que asumen estas redes es conducir el proceso de transformación que permita pasar de una oferta de formación técnica desarticulada y, en muchos casos, descontextualizada de las necesidades del país, hacia una articulada, que responda a las demandas del desarrollo productivo.

La red, al estar conformada por agentes productivos y formativos en torno a un sector determinado, debería constituirse además en agente dinamizador del desarrollo productivo en sus respectivas regiones, que prepare y actualice, en forma permanente y pertinente, los cuadros técnicos que dicho sector requiera para enfrentar competitivamente los desafíos que impone una economía globalizada.

Este nuevo valor que las redes concederán al sistema deberá ser internalizado por los actores que las conforman (los agentes formadores y capacitadores, las empresas y las organizaciones de trabajadores), para transformarlo en una oferta concreta y accesible, que sea percibida y asumida por cada persona como una herramienta que contribuye efectivamente al desarrollo de sus capacidades, empleabilidad y expectativas de progreso personal.

Actualmente, en las 25 redes que ejecutan proyectos para articulación de la formación técnica en las trece regiones del país, están involucradas ${ }^{1} 54$ universidades, 72 centros de formación técnica (CFT) e institutos profesionales (IP), 232 liceos de enseñanza media técnico-profesional, 49 organismos técnicos ejecutores de capacitación (OTEC), dos instituciones de educación de adultos y 133 del sector productivo y de organizaciones de trabajadores.

Partiendo de la base que la asociación en red es una organización compleja, ha interesado principalmente que el funcionamiento de cada una de las redes sea consecuente con el propósito central del

1 En estas cifras se debe considerar que algunas universidades y CFT integran más de una red. Por ejemplo, la Universidad de Talca participa en las redes de la Madera, Hortifrutícola y Vitivinícola de su región; la Universidad de Concepción en las redes de Metalmecánica, Madera y Agroindustrial. 
Programa Chilecalifica y que contribuya a abordar los temas de mejoramiento de la calidad de la formación técnica, de su articulación curricular y de sus relaciones con el desarrollo productivo regional, con las organizaciones productivas y de trabajo.

En una visión sistémica del concepto de red, Del Valle (2003) expresa que: "La red es un tipo particular de sistema social, que se caracteriza por una asociación voluntaria de personas o de instituciones (los participantes), para el logro de un propósito o finalidad común. Tal asociación sólo será viable si posee la propiedad central de sinergia, que se puede especificar a través de dos condiciones simultáneas:

- La red avanzará hacia el logro de su propósito si contribuye al logro de los fines de sus participantes.

- Los participantes avanzarán hacia el logro de sus propios fines si contribuyen al logro del propósito de la red".

El autor señala que “...si estas condiciones no se cumplen, los participantes perderán interés en la red y ésta dejará de tener viabilidad. Si sólo se cumplen para algunos participantes, los demás se sentirán desplazados o utilizados y se generarán tensiones que pondrán su factibilidad en peligro. Algo similar ocurrirá si hay participantes que dejan de contribuir lo que se espera de ellos y sólo buscan aprovecharse de la acción de los demás; estos últimos tenderán a expulsarlos de la red. Por otra parte, con una perspectiva de más largo plazo, toda red aspirará a que su viabilidad sea permanente y se fundamente en el logro del propósito común que, en este caso, es construir una oferta articulada de formación técnica en la región y el sector respectivo. En la medida en que este propósito se logre, la red podrá captar valor y alcanzar un adecuado nivel de sustentabilidad".

En este sentido, para lograr una verdadera articulación entre las instituciones que conforman la red, se debe asumir que los intereses particulares de cada una de las instituciones no son contrapuestos o excluyentes con los intereses de las demás, sino que, por el contrario, son compatibles y complementarios. 
Entre los beneficios que los diferentes actores pueden esperar de esta asociación en red, se pueden mencionar los siguientes:

- Beneficios para empresarios y trabajadores al participar en la red Empresarios

- Técnicos formados de acuerdo a las competencias laborales definidas por la empresa.

- Ahorro en acciones de capacitación e inducción de los trabajadores que se incorporan a la empresa.

- Capacitación y actualización permanente de los trabajadores de acuerdo a sus definiciones.

- Socios formadores con mayor capacidad de respuesta a los nuevos requerimientos de formación y capacitación de los trabajadores.

- Generación de nuevas alianzas productivas.

- Apoyo directo en procesos de certificación de competencias de los trabajadores.

Trabajadores

- Mejoramiento de capacidades y empleabilidad.

- Posibilidades de acceso a certificación de competencias laborales.

- Mayor acceso a la capacitación pertinente.

- Mejoramiento de las expectativas laborales y de desarrollo profesional y personal.

- Acceso a la obtención de títulos técnicos a través del reconocimiento de su experiencia laboral y competencias adquiridas a través de la capacitación.

- Beneficios para instituciones formadoras al participar en la red Universidades, IP y CFT

- Aumento de cobertura de la formación técnica.

- Establecimiento de alianzas con otras entidades formativas.

- Mejoramiento de la calidad y pertinencia de su oferta curricular.

- Actualización permanente de su oferta formativa.

- Desarrollo de planes de actualización de docentes técnicos. 
- Vínculo directo con la realidad productiva (tendencias y tecnología).

- Investigación y desarrollo tecnológico.

Liceos EMTP, Educación de Adultos y OTEC

- Mayor pertinencia de su oferta curricular.

- Mejoramiento del proceso formativo por mayor acceso (compartido) a recursos tecnológicos.

- Mejores expectativas laborales para sus egresados.

- Mayores posibilidades de continuidad de estudio de sus egresados.

- Capacitación y actualización de docentes técnicos acorde a las demandas del sector productivo.

- Acceso a pasantía y prácticas profesionales de alumnos en empresas.

Si bien son destacables los avances en materia de organización interna de la redes, este proceso no ha dejado de presentar dificultades. Una de las mayores trabas en el proceso de gestión de la red y en la puesta en marcha de sus proyectos ha sido, por una parte, el cambio cultural que implica la asociación de instituciones formativas y productivas y, por otra, el trabajo conjunto de instituciones con modelos de gestión y organización tan diversos.

Otra de las dificultades ha sido la tendencia de algunas instituciones formativas, representantes de las redes, que - por su historia, trayectoria y posicionamiento en la región- tienden a limitar significativamente los niveles de participación de otros integrantes, tanto en la gestión de la red propiamente tal como en la ejecución del proyecto. Esto ha implicado una desmotivación de las instituciones que quedan excluidas de las decisiones, lo cual se ha traducido en la disminución sustantiva de cooperación y participación en las acciones impulsadas por la red.

Por esta razón, se están diseñando, en conjunto con las autoridades y equipos regionales del programa, estrategias para el fortalecimiento de mecanismos de participación al interior de 
las redes y el desarrollo de acciones conjuntas que favorezcan su posicionamiento regional. Obviamente, ninguna red es igual a otra. Son diferentes por su conformación, por las personas que participan en representación de cada institución, por el tamaño y número de las instituciones que las conforman, por el impacto que ellas pueden generar en el desarrollo productivo o formativo de la región, por el entorno en que operan y por las metas específicas que se proponen alcanzar.

Por esto, una de las tareas que se ha propuesto el programa es conocer en profundidad las características de asociatividad y vinculación que cada una de las redes de articulación de la formación técnica ha generado desde su conformación, con el fin de entregar elementos que permitan mejorar y potenciar la interacción y su gestión interna.

Si bien es cierto, al momento de conformar las redes, una de las primeras reticencias que tenían los representantes de las instituciones apuntaba a dudar del beneficio que obtendrían de estas nuevas alianzas, resultó estimulante ver cómo, a los pocos meses, en algunas redes, el foco de la discusión cambió hacia la forma en que cada una podía contribuir al logro de los objetivos comunes, dando respuesta de una forma curiosa a la pregunta inicial: la contribución de cada institución es el beneficio de las demás. Si todas contribuyen, todas ganan.

Entre las contribuciones que las empresas y el sector productivo han venido comprometiendo para mejorar los procesos formativos, se señalan:

- Orientación y apoyo a la gestión interna de las redes.

- Definición de prioridades y tendencias del desarrollo productivo regional.

- Definición de competencias laborales y perfiles profesionales.

- Establecimiento de necesidades de capacitación y actualización de trabajadores y técnicos.

- Oferta de espacios para formación por alternancia, prácticas y pasantías para alumnos y docentes en la empresa.

- Acceso a nuevas tecnologías productivas. 
Por su parte, entre las contribuciones de las instituciones formativas y de capacitación a los procesos productivos, se mencionan:

- Oferta formativa pertinente a las necesidades del desarrollo productivo regional.

- Formación de técnicos enfocados a las competencias laborales.

- Desarrollo de oferta de capacitación modularizada y articulada con la oferta educativa formal.

- Desarrollo de investigaciones tecnológicas para potenciar el desarrollo productivo regional.

- Actualización de los docentes para el mejoramiento de la formación de técnicos en los diferentes niveles.

- Evaluación de competencias y reconocimiento de aprendizajes previos de los trabajadores.

\section{El nuevo foco del tercer concurso de proyectos}

A partir de las experiencias recogidas en las redes que llevan más de un año ejecutando sus proyectos y de la evaluación realizada a los procesos de gestión y ejecución, se ha rediseñado la estrategia para el tercer concurso, orientando los proyectos fundamentalmente a la implementación de propuestas curriculares con enfoque de competencia laborales.

Con esta orientación, se cambia el énfasis de los proyectos de articulación, concentrando los esfuerzos en la implementación de los diseños curriculares en los sectores productivos abordados por los proyectos, de modo que el beneficio —alcanzar mayores niveles de pertinencia y calidad en la formación de técnicos- se encuentre efectivamente a disposición de las personas a partir del año 2008, dándoles la posibilidad de que se les reconozcan sus aprendizajes previos y se le presenten diversas posibilidades para continuar estudios superiores.

La convocatoria del tercer concurso de redes se realizará durante 2005. Se espera que todas las universidades que imparten 
formación técnica, además de los CFT e IP, se involucren en la conformación de redes, en conjunto con los liceos, instituciones de capacitación y el sector productivo.

Considerando los avances experimentados por las diferentes redes en la elaboración de las propuestas formativas para diversos sectores productivos y la articulación de este tipo de propuestas formativas encargadas por el Programa Chilecalifica a diversas instituciones de enseñanza superior, se ha estimado pertinente profundizar, en este tercer concurso, en aspectos complementarios requeridos para una adecuada implementación de dichas propuestas.

En función de lo expuesto, las redes que resulten seleccionadas en el tercer concurso de proyectos deberán abordar, al menos, las siguientes tareas:

- Implementar itinerarios de formación técnica, garantizando su pertinencia de acuerdo con los requerimientos del sector productivo regional correspondiente.

- Desarrollar modelos didácticos y evaluativos que permitan la adecuada y eficiente implementación de las propuestas formativas.

- Desarrollar las acciones necesarias de actualización docente para que las instituciones cuenten con una adecuada conducción de los procesos formativos.

- Establecer los mecanismos necesarios de reconocimiento de aprendizaje para la incorporación de personas que cuenten con formación o aprendizajes previos que ameriten ser reconocidos.

Las propuestas deberán constituir experiencias concretas de implementación curricular en la formación técnica que se enmarquen en la perspectiva de Sistema de Formación Permanente, debiendo conectarse con los requerimientos que imponga el Sistema Nacional de Certificación de Competencias Laborales cuando empiece a operar. 
Con la ejecución de la totalidad de los proyectos que financiará el Programa Chilecalifica, se completan las experiencias demostrativas en los diversos sectores productivos y regiones del país. Con ello, se espera dejar instaladas las bases para el funcionamiento de un sistema de formación permanente, a través del cual las personas o alumnos tengan acceso a una oferta de formación técnica integrada, que incluya al menos:

- Formación en oficios para personas adultas que nivelan educación básica.

- Formación de técnicos de nivel medio, para jóvenes y adultos, en la enseñanza media técnico-profesional.

- Formación de técnicos de nivel superior, en educación superior.

- Posibilidad de continuidad de estudios superiores.

- Capacitación laboral articulada con ofertas formales.

- Reconocimiento de aprendizajes para establecer sus propias trayectorias formativas.

Esta es la forma en que el programa Chilecalifica, a través de la estrategia de redes, pretende contribuir al mejoramiento de la calidad y pertinencia de la formación técnica en nuestro país, y generar un sistema de formación permanente que permita a las personas la actualización continua de sus competencias y mantener vigente su empleabilidad.

El logro de estos objetivos no es sólo responsabilidad de un programa, es una tarea del país que convoca a todos los actores involucrados en la definición e implementación de propuestas de formación y capacitación. La experiencia y capacidades de las instituciones de educación superior constituyen un aporte fundamental para el logro de los objetivos expuestos, en la medida en que se logren generar dinámicas colaborativas al interior de las redes, se compartan las responsabilidades y las decisiones y se diluyan los protagonismos institucionales. 


\section{Referencias bibliográficas}

Brunner, José Joaquín. Educación e Internet: ¿La próxima revolución? Fondo de Cultura Económica, Santiago de Chile, 2003.

Brunner, José Joaquín. El nuevo pluralismo educacional en América Latina. FLACSO, Santiago de Chile, 2003.

Irigoin, M. Etiennette. Hacia una educación permanente en Chile. Santiago de Chile, CEPAL, 2002.

Del Valle, Alfredo. El ETP: Una herramienta en Internet para comunicación y gestión del conocimiento. En: Minerales, v. 58, n. 249, Instituto de Ingenieros de Minas de Chile, enero-febrero 2003. 